\title{
Electrochemical deposition of aniline derivatives for conductometric gas sensors
}

\author{
M. Mateos ${ }^{\mathrm{a}, *}$, R. Meunier Prest ${ }^{\mathrm{a}}, \mathrm{JM}_{\text {. Suisse }}^{\mathrm{a}}$, M. Bouvet ${ }^{\mathrm{a}}$ \\ anstitut de Chimie Moléculaire de l'Université de Bourgogne, CNRS UMR 6302, Univ. Bourgogne Franche-Comté, 21078 Dijon, France
}

\begin{abstract}
Polymer film of poly(2,3,5,6-tetrafluoroaniline) (PTFA) were electroplated on ITO substrate from acidic medium by chronoamperometry. Electrochemical and morphological characterizations were performed and compared to polyaniline properties similarly coated. It seemed that PTFA film had an irreversible redox response with poor conductivity due to the absence of acid-base doping. This film were then incorporated in a patented device called MSDI heterojunction to perform ammonia sensing in humid atmosphere.
\end{abstract}

Selection and/or Peer-review under responsibility of SURFOCAP 2017.

Keywords: ammonia, heterojunction, conductometric sensors, polyaniline,

\section{Introduction}

Widely used in the field of organic electronics and sensors, conducting polymers are the object of intensive research due to their great chemical variety and their processability by electropolymerization [1]. The electronic properties of polyaniline derivatives are highly dependent on the nature of the substituents on the aniline ring. Numerous studies have focused on the properties of monofluorinated derivatives [2,3], but only a few on the fully fluorinated polyaniline (PolyTetraFluoroAniline, PTFA) [4]. In this study, we present electrochemical and morphological properties of PTFA polymer electroplated on conductive substrates, in comparison with polyaniline already used as sensing material in $\mathrm{NH}_{3}$ sensors $[5,6]$. Finally, this polymer was incorporated into a patented device called Molecular Semiconductor - Doped Insulator (MSDI) heterojunction [7] that combined a poor conductive material such as monophtalocyanines or perylene and an intrinsic molecular semiconductor, the lutetium bisphtalocyanine $\left(\mathrm{LuPc}_{2}\right)$.

\section{Experimental}

2,3,5,6-terafluoroaniline (TFA, Aldrich) was used as received. Ammonia gas, $1000 \mathrm{ppm}$ in synthetic air, and synthetic air were used from standard cylinders, purchased from Air Liquide, France. Lutetium bisphtalocyanine $\left(\mathrm{LuPc}_{2}\right)$ were synthesized according to previously described methods.

All electrochemical experiments were performed with a PGSTAT302 N (Metrohm) potentiostat connected to a PC and the data collected were analyzed using Nova ${ }^{\circledR} 2.1$ Software. Cyclic Voltammetry (CV) and

\footnotetext{
* Corresponding author. Tel.: +0-000-000-0000 ; fax: +0-000-000-0000 .

E-mail address: mickael.mateos@u-bourgogne.fr
} 
Chronoamperometry (ChA) was carried out by means of a three-electrode configuration consisting of a carbon disk (3 mm diameter, Bioanalytical Systems) or Indium Tin Oxide (ITO) plate (9 mm diameter of active surface, 8-12 $\Omega$ of square resistance, Solems, Palaiseau, France) or ITO interdigitated electrodes (IDE, deposited onto a $1 \mathrm{x} 1 \mathrm{~cm}^{2}$ glass substrate and separated by $75 \mu \mathrm{m}$ with $50 \mathrm{~nm}$ thickness) as working electrode, a platinum wire as counter electrode and a saturated calomel electrode (SCE) as reference electrode. Potentials were reported versus SCE.

SEM imaging was performed with scanning electronic microscope JEOL JSM6400F with an acceleration voltage of $2 \mathrm{kV}$. XPS analysis of the PTFA powder and polymer films deposited on ITO was performed on a SIA100 spectrometer (Cameca Riber apparatus) using non-monochromated Al K $\alpha$ X-ray source (1486.6 eV photons). PANI films were deposed on ITO electrodes by chronoamperometry at $1 \mathrm{~V}$ in a solution of aniline $0.15 \mathrm{M}^{\text {in }} \mathrm{HClO}_{4} 2 \mathrm{M}$ with a surface charge of $265 \mathrm{mC} . \mathrm{cm}^{-2}$, then rinced with $\mathrm{HClO}_{4} 0.1 \mathrm{M}$, absolute $\mathrm{EtOH}$ and dried under vacuum at room temperature. PTFA films were prepared by chronoamperometry at $1.4 \mathrm{~V}$ in a solution of TFA $50 \mathrm{mM}$ in $\mathrm{HClO}_{4}$ $2 \mathrm{M}$ The electrolysis was stopped after consumption of $250 \mathrm{mC} . \mathrm{cm}^{-2}$. The modified electrode was rinsed in $\mathrm{HClO}_{4}$ $0.1 \mathrm{M}$ and dried under vacuum at room temperature.

Electrical measurements were carried out with IDE. First, a PTFA bottom layer (thickness $1 \mu \mathrm{m})$ was electrodeposited on both IDE by chronoamperometry at $1.4 \mathrm{~V}$ (surface charge of $250 \mathrm{mC} . \mathrm{cm}^{-2}$ ) in a solution of 50 $\mathrm{mM}$ TFA in $\mathrm{HClO}_{4} 2 \mathrm{M}$. Then, the top layer was coated by sublimation of the bisphtalocyanine of lutetium $\left(\mathrm{LuPc}_{2}\right)$ in an UNIVEX 250 thermal evaporator (Oerlikon, Germany), under secondary vacuum (ca. $10^{-6} \mathrm{mbar}$ ), at a rate of 1 $\AA . s^{-1}$, by heating in a temperature range of $400-500{ }^{\circ} \mathrm{C}$. The workbench used for $\mathrm{NH}_{3}$ exposure, at different relative humidity (rh) values, was described previously [8]. The total flow was in the range $0.5-0.55 \mathrm{~mL} \cdot \mathrm{min}^{-1}$ depending on ammonia concentration and the volume of the test chamber was $8 \mathrm{~cm}^{3}$. Gas sensing experiments were carried out in a dynamic way, with 4 min-long rest periods alternating with 1 min-long exposure periods.

\section{Result and discussion}

\subsection{Electrochemical characterization of the PTFA polymer}

Firstly, a CV of the monomer (TFA) in an acid medium was carried out on a carbon electrode (see Fig. 1a). The voltamperogram shows an important peak of oxidation at $1.2 \mathrm{~V}$ which is weakened over the cycles, attributed to the oxidation of the monomer during the polymerization mechanism. The redox system centered at $0.6 \mathrm{~V}$ and becoming more and more irreversible over the cycles, corresponds to the polymer system (passage from the amine form to the imine). The increase of the irreversibility is due to the passivation of the active surface of the electrode by the polymer.

The Fig. $1 \mathrm{~b}$ shows the electrochemical response of a $1 \mu \mathrm{m}$ film of PANI (green) and PTFA (red) electrodeposited on an ITO substrate respectively at $1 \mathrm{~V}$ (charge surface of $265 \mathrm{mC} . \mathrm{cm}^{-2}$ ) and $1.4 \mathrm{~V} 250 \mathrm{mC} . \mathrm{cm}^{-2}$ ). As expected for the PANI film, we notice two redox systems with a high current range $(1 \mathrm{~mA})$. The first one slightly irreversible $\left(\mathrm{E}_{\mathrm{red}}=0.05 \mathrm{~V}\right.$ and $\left.\mathrm{E}_{\mathrm{ox}}=0.34 \mathrm{~V}\right)$ corresponds to the passage from the leucoemeraldine to the emeraldine state and the second reversible system $\left(\mathrm{E}_{\mathrm{red}}=0.52 \mathrm{~V}\right.$ and $\left.\mathrm{E}_{\mathrm{ox}}=0.62 \mathrm{~V}\right)$ corresponds to the transition from the emeraldine to the pernigraniline state. While, we only observe an strongly irreversible system for PTFA $\left(\mathrm{E}_{\mathrm{red}}=0.02 \mathrm{~V}\right.$ and $\mathrm{E}_{\mathrm{ox}}=$ $1.38 \mathrm{~V})$ with a lower current range $(30 \mu \mathrm{A})$ with a large shift of the anodic peak due to the fluorination of the aniline ring which make the oxidation more difficult. 

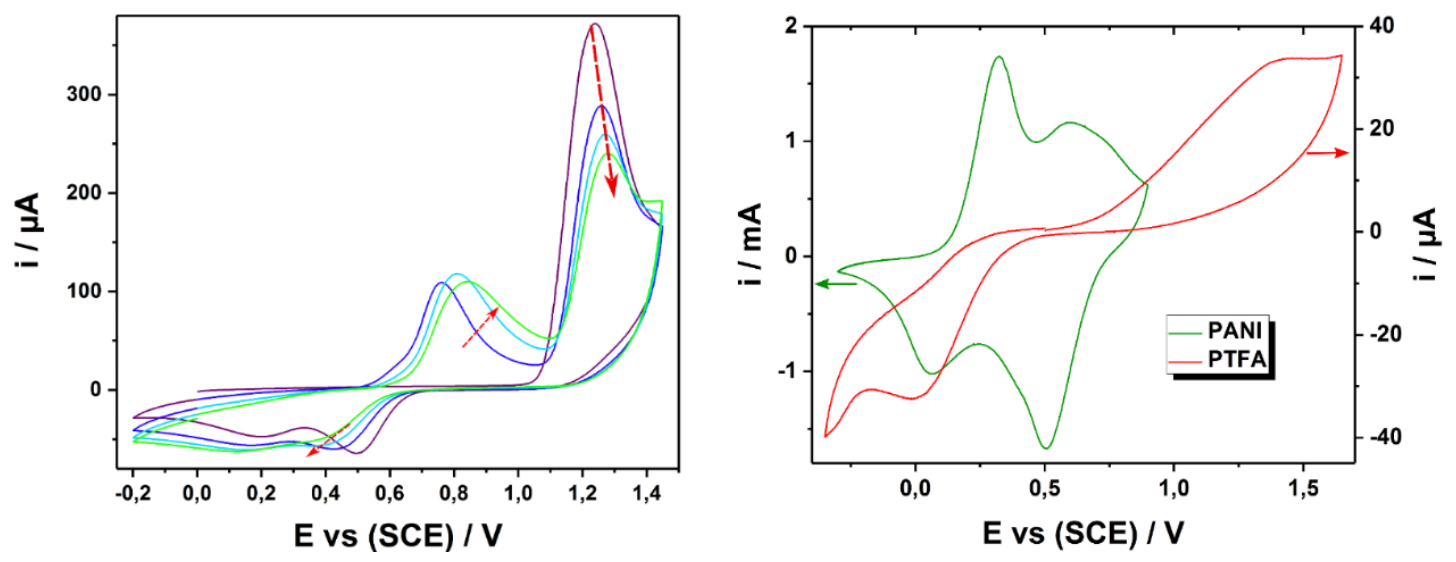

Fig. 1: (a) Cyclic voltammetry on glassy carbon of TFA $30 \mathrm{mM}$ in $\mathrm{HClO}_{4} 0.66 \mathrm{M}, \mathrm{v}=0.1 \mathrm{~V} \cdot \mathrm{s}^{-1}$; (b) Cyclic voltammetry PTFA and PANI polymer, coated on ITO, in $\mathrm{HClO}_{4} 0.5 \mathrm{M}, \mathrm{v}=0.04 \mathrm{~V} . \mathrm{s}^{-1}$

\subsection{SEM images and XPS analysis of coated PTFA and PANI}

SEM imaging revealed a profound difference in morphology between PTFA and PANI layers. The growth of PTFA on ITO is slow (strong decrease of the current during the ChA) because this fluorinated polymer has a little affinity for the surface and its low conductivity limits its growth. Then, a very compact layer is obtained with the presence of amorphous grains (see Fig. 2b). While, the PANI layer has a rapid growth (increase of the current during the ChA after passing through a minimum) in the form of fibers because the high conductivity of the polymer in its doped form allows it to rapidly grow on itself (see Fig. 2c).
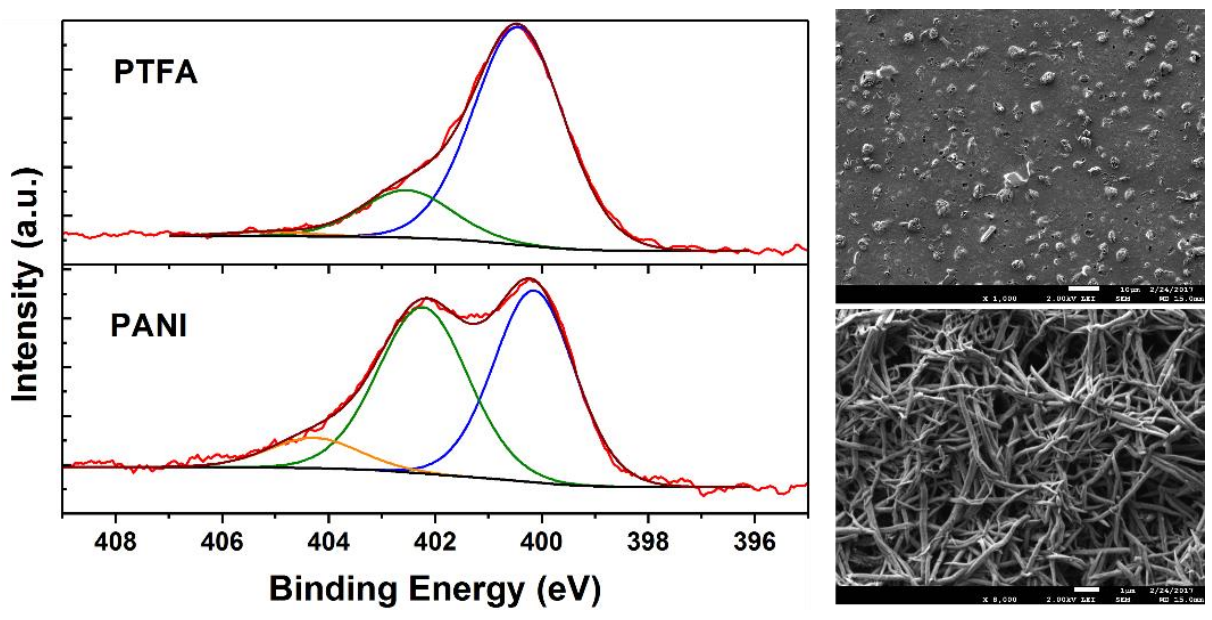

Fig. 2: (a) N1s spectra of PTFA and PANI layers coated on ITO substrate by chronoamperometry ; (b) SEM images of PANI and (c) PTFA layers coated on ITO substrate.

The atomic ratio (nitrogen used as reference) of $\mathrm{C}, \mathrm{N}, \mathrm{O}, \mathrm{F}$ and $\mathrm{Cl}$ has been calculated using peaks area of the different elements for each polymer films. These results are in a good agreement for PANI, $5.7 \mathrm{C}$ for $1 \mathrm{~N}$ with a high chlorine level (0.8) that revealed an important acid-base doping (protonation of imine) due to the presence of $\mathrm{ClO}_{4}{ }^{-}$. However, for PTFA the results indicated a carbon pollution (7.7) and a lack of fluorine (2.7). Moreover, we notice a low level of chlorine (0.2) that indicated the absence of doping. The analysis of N1s spectra of PANI (see Fig. 2a) revealed three different form: the imine at $400.1 \mathrm{eV}$, the amine at $402.2 \mathrm{eV}$ and the protonated imine at 
$404.3 \mathrm{eV}$ that confirmed the acid-base doping. We also found the imine and amine form for PTFA at 400.4 and $402.5 \mathrm{eV}$ but the protonated imine form at $405.2 \mathrm{eV}$ was very weak that confirmed the absence of doping.

\subsection{I(V) Characteristics and electrical response under ammonia and humidity}

Electrical measurements in ambient atmosphere of $\mathrm{LuPc}_{2}$ resistor $(50 \mathrm{~nm})$ coated by sublimation on IDE (see Fig. $3 \mathrm{a}$ in blue) showed a linear $\mathrm{I}(\mathrm{V})$ characteristics in accordance with an ohmic behavior. However the characteristic of the MSDI heterojunction made with PTFA film $(1 \mu \mathrm{m})$ as bottom layer and $\operatorname{LuPc}_{2}(50 \mathrm{~nm})$ as top layer revealed a non-linearity at low bias. This typical behavior can be explained by the formation of an energy barrier caused by the difficulty for the electrons to cross both the bottom layer and the heterojunction between the two layers.

The MSDI device was then used as an ammonia sensor (see Fig. 3a) in a humid atmosphere (from $70 \%$ to $10 \%$ of relative humidity). We noticed a decrease of the current during the $\mathrm{NH}_{3}$ exposition phase (from $90 \mathrm{ppm}$ to $30 \mathrm{ppm}$ ) at any relative humidity that demonstrate the ability of $\mathrm{NH}_{3}$ detection in humid atmosphere. However, the relative change of the current remained weak in the $\mathrm{NH}_{3}$ range used that indicated the possible overload of the sensor. We can also conclude the $\mathrm{p}$ type character of the PTFA film because we observed that a reductive gas such as $\mathrm{NH}_{3}$ involved a drop of the conductivity of the device.
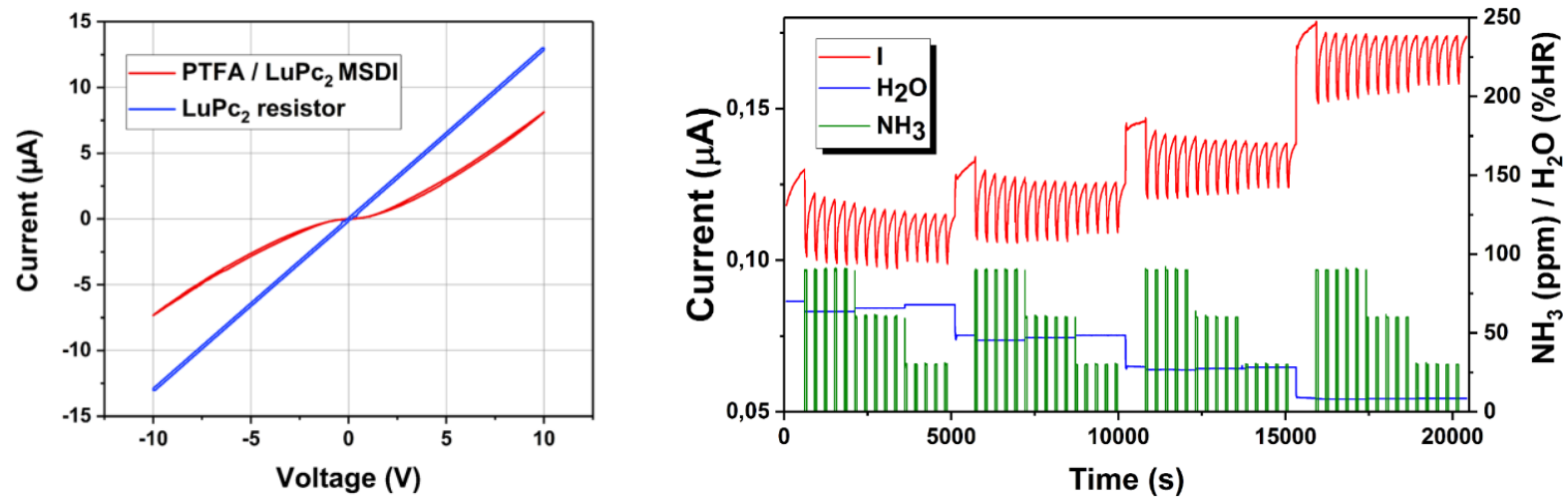

Fig. 3: (a) Current-voltage characteristics of a LuPc 2 resistor $(50 \mathrm{~nm}$, blue) and a PTFA ( 1 $\mu \mathrm{m}) / \mathrm{LuPc}_{2}(50 \mathrm{~nm}) \mathrm{MSDI}$ (red); (b) Response of a PTFA / $\mathrm{LuPc}_{2}$ MSDI polarized at $1.5 \mathrm{~V}$ depending on $\mathrm{NH}_{3}(\mathrm{~g})(\mathrm{ppm})$ and relative humidity $(\% \mathrm{HR})$

\section{Conclusion and perspectives}

We demonstrated that a fully fluorinated polyaniline has different properties than its unsubstituted analogue. It is an undoped dense polymer with a non-measurable conductivity. Its use in an MSDI heterojunction device confirmed its $\mathrm{p}$ type character despite the full fluorination, without demonstrating a higher relevance compared to copper perfluorophthalocyanine $\left(\mathrm{CuF}_{16} \mathrm{Pc}\right)$ usually used in n-MSDI heterojunction [7].

\section{Acknowledgements}

The authors acknowledge the ANR for funding through the projects CAP-BTX 2010 and OUTSMART 2015, the Conseil Régional de Bourgogne (projects FABER, PARI SMT08 and CDEA), the European Union (FEDER and Cost action TD1105 EuNetAir) and finally the MENESR for funding the PhD grant (M. M.).

\section{References}

[1] A.G. MacDiarmid, Angew. Chem. Int. Ed. 40 (2001) 2581-2590.

[2] A.L. Sharma, M. Gerard, R. Singhal, B.D. Malhotra, S. Annapoorni, , Appl. Biochem. Biotechnol. 96 (2001) $155-165$.

[3] A. Cihaner, A.M. Önal, Polym. Int. 51 (2002) 680-686.

[4] L. Astratine, E. Magner, J. Cassidy, A. Betts, Electrochimica Acta. 74 (2012) 117-122. 
[5] H. Liu, J. Kameoka, D.A. Czaplewski, H.G. Craighead, Nano Lett. 4 (2004) 671-675.

[6] D. Nicolas-Debarnot, F. Poncin-Epaillard, Anal. Chim. Acta. 475 (2003) 1-15.

[7] V. Parra, J. Brunet, A. Pauly, M. Bouvet, Analyst. 134 (2009) 1776-1778.

[8] P. Gaudillat, A. Wannebroucq, J.-M. Suisse, M. Bouvet, Sens. Actuators B Chem. 222 (2016) 910-917. 